\title{
On the chirality of torus curves and knots
}

\author{
Wagnière, Georges H
}

\begin{abstract}
As is well known, a (p, q) torus knot is topologically equivalent to a (q, p) torus knot. The sign of the writhe number, which characterizes the topological chirality, must evidently be the same in both cases. We here show by an analytic criterion related to the torsion that a $(\mathrm{p}, \mathrm{q})$ torus curve and a (q, p) torus curve have opposite chirality, although they are not enantiomers
\end{abstract}

DOI: https://doi.org/10.1007/s10910-006-9086-9

Posted at the Zurich Open Repository and Archive, University of Zurich ZORA URL: https://doi.org/10.5167/uzh-155723

Journal Article

Published Version

Originally published at:

Wagnière, Georges H (2007). On the chirality of torus curves and knots. Journal of mathematical chemistry, 41(1):27-31.

DOI: https://doi.org/10.1007/s10910-006-9086-9 


\title{
On the chirality of torus curves and knots
}

\author{
Georges H. Wagnière \\ Institute of Physical Chemistry, University of Zurich, Winterthurerstr. 190, \\ CH-8057 Zurich, Switzerland \\ E-mail: wagniere@pci.unizh.ch
}

\begin{abstract}
As is well known, a $(p, q)$ torus knot is topologically equivalent to a $(q, p)$ torus knot. The sign of the writhe number, which characterizes the topological chirality, must evidently be the same in both cases. We here show by an analytic criterion related to the torsion that a $(p, q)$ torus curve and a $(q, p)$ torus curve have opposite chirality, although they are not enantiomers.
\end{abstract}

KEY WORDS: torus curves, torus knots, chirality, torsion

\section{Introduction}

There have been various attempts to quantitatively measure chirality in the frame of enantioselective stereochemistry [1-3]. In recent years molecules have been discovered or synthesized that form knots and links [4-6]. In such cases one also encounters topological chirality. The topological chirality of knots and links has been the subject of extensive investigations [7-9]. Topological chirality is also an important criterion in the hydrodynamic description of vortices and in interpreting magnetohydrodynamic phenomena [10-12].

Among simple knots, torus knots play a particular role, as they can be created by closed curves on the surface of a torus. We wish to show by this example that the topological chirality and the geometric chirality of a torus curve do not necessarily coincide, and that the two different curves belonging to one and the same knot will even have opposite geometric chirality. The conclusion to be drawn therefrom is that the chirality of a space curve-for instance as representative of a molecular strand - must essentially be based on criteria of analytic geometry, no matter if it forms a knot or not. If one is interested in molecular electronic and spectroscopic properties, such as CD spectra, one is in any case obliged to base theoretical predictions on a detailed geometric structure, and purely topological criteria are insufficient.

The parametric equations for torus curves in $3 \mathrm{D}$, which may be immediately derived from the equations for the surface of a torus, read: 


$$
\begin{aligned}
& x(t)=(a+r \cos p t) \cos q t, \\
& y(t)=(a+r \cos p t) \sin q t, \\
& z(t)=r \sin p t .
\end{aligned}
$$

$p, q$ are coprime integers (with only 1 as common divisor), $t$ is an angular parameter. The constant integer $p$ refers to the meridian angle, with corresponding tube radius $r$; the constant integer $q$ refers to the longitudinal angle (around the axis going through the center of the hole of the torus), with corresponding torus radius $a$. In general, one assumes $a \gg r$. For $t$ going from 0 to $2 \pi$, these equations describe closed curves. Many of these curves display overcrossings and undercrossings and therefore topologically correspond to knots. As is well known [13], the minimal number of crossings $c$ of a $(p, q)$ torus curve or knot is given by:

$$
c=\min \{p(q-1), q(p-1)\}
$$

This marks the fact that a $(p, q)$ torus knot is topologically equivalent to a $(q, p)$ torus knot, where in the second bracket $q$ refers to the meridian angle and $p$ to the longitudinal angle. As an example, both $(3,2)$ and $(2,3)$ stand for the trefoil knot.

\section{The chirality of torus curves}

We now consider torus curves from a geometric point of view. Elementary differential geometry teaches us that space curves in 3D are characterized by three quantities, the arc length, the curvature and the torsion. While the first two quantities are insensitive to chirality, the torsion $\tau$, however, changes sign on going from a right-handed to a left-handed coordinate system. If the space curve is expressed in the parametric form $x(t), y(t), z(t)$, then the torsion $\tau$ is given by [14]:

$$
\tau(t)=\frac{A(t)}{B(t)},
$$

where

$$
A(t)=\left|\begin{array}{ccc}
\dot{x} & \dot{y} & \dot{z} \\
\ddot{x} & \ddot{y} & \ddot{z} \\
\dddot{x} & \dddot{y} & \dddot{z}
\end{array}\right|
$$

and

$$
B(t)=(\dot{x} \ddot{y}-\dot{y} \ddot{x})^{2}+(\dot{y} \ddot{z}-\dot{z} \ddot{y})^{2}+(\dot{z} \ddot{x}-\dot{x} \ddot{z})^{2},
$$


with $\dot{x}=\frac{\partial x}{\partial t}, \ddot{x}=\frac{\partial^{2} x}{\partial t^{2}}$, etc. It is immediately apparent that the determinant $A(t)$ is a pseudoscalar quantity and that therefore it is odd under the operation of parity. Thus $A(t)$ must have opposite sign for space curves that are chiral and enantiomeric. In contrast, the denominator $B(t)$ is a true parity-even scalar. In what immediately follows, we thus focus our attention mainly on $A(t)$ as a characteristic, chirality-specific quantity. For any chiral segment of a space curve $\left(t_{1}-t_{2}\right)$ we should find:

$$
\int_{t_{1}}^{t_{2}} A(t) \mathrm{d} t \neq 0
$$

For closed torus curves $(p, q)$ this will be the case in the limits 0 to $2 \pi$. The corresponding integration of (3a) is somewhat lengthy, but may be straightforwardly carried out with a program such as Maple. It leads to a surprisingly simple result:

$$
I_{A}(p, q)=\int_{0}^{2 \pi} A(p, q ; t) \mathrm{d} t=2 p q\left(q^{4}-p^{4}\right) a \pi
$$

( $r$ in (1) is here for simplicity set $=1$, and $a$ is thus equal to the proportion of the torus radius to the tube radius.) We immediately notice that an interchange of $p$ and $q$ leads to a change of sign of the integral. This implies that a $(p, q)$ torus curve and a $(q, p)$ torus curve should have opposite geometric chirality, although they are definitely not true enantiomers. On the other hand, the corresponding knots, when they exist, are topologically equivalent and must thus display the same topological chirality. For instance, assuming $a=3$, we find for the trefoil knot:

$I_{A}(3,2)=-2340 \pi$, and $I_{A}(2,3)=+2340 \pi$, but the sign of the writhe number is the same in both cases. A computer visualization of torus curves and knots demonstrates this very instructively $[15,16]$. In general, a $(p, 1)$ curve corresponds to a left-handed (meridian) toroidal helix, while a $(1, q)$ curve appears as a (longitudinal) double spiral. The outer part of the spiral has a right-handed twist, the inner part is left-handed. Obviously, the right-handed part dominates the integrated chirality. Similar observations are made for the knots $(p, 2)$ and $(2, q)$, etc. From (5) we also conclude that the chirality integral $I_{A}(p, q)$ vanishes if $p=q$, or if either $p$ (and) or $q$ are zero. Visualization [15] again confirms this. It is furthermore found that if $(p, q)$ are coprime, then the curve $(n p, n q)$, where $n$ is an integer, does not lead to an independent new curve; rather the curve $(n p, n q)$ is geometrically equivalent to $(p, q)$. Introducing the condition into (5), we notice that the quantity $n^{6}$ may be factored out and cancelled against the same factor in the integrated denominator $B(t)$. If we separately integrate the denominator $B(t)$ from 0 to $2 \pi$, we also obtain a polynomial of order 6 in $p, q$, but containing only the even exponents $p^{6}, p^{4} q^{2}, p^{2} q^{4}$ and $q^{6}$. 
A more comprehensive investigation of the chirality of space curves undoubtedly requires an examination of the torsion $\tau(t)$ itself, and not only of the parity-odd numerator $A(t)$. For torus curves, a closed-form integration

$$
I_{\tau}(p, q)=\int_{0}^{2 \pi} \tau(p, q ; t) \mathrm{d} t .
$$

It does not appear to be straightforwardly feasible, however, and probably requires numerical integration. One will conceivably find that $I_{\tau}(p, q)$ and $I_{\tau}(q, p)$ have opposite sign, but very probably a different absolute value. The torus curves $(p, q)$ and $(q, p)$ are, as mentioned, not true enantiomers. True enantiomers of $(p, q)$ are given by the curves $(-p, q)$ or $(p,-q)$. The former curve corresponds to a reflection of the curve $(p, q)$ in the $x, y$-plane, the latter to a reflection in the $x, z$-plane. It is of course evident that:

$$
I_{\tau}(p, q)=-I_{\tau}(-p, q)=-I_{\tau}(p,-q) .
$$

The same holds for $I_{A}(p, q)$, but this is trivial.

\section{Chemical and physical significance}

Torus curves are a good example to illustrate the difference between topological chirality and geometric chirality. Their interest not only lies in the fact that they form an important class of knots, but also that they are of chemical and physical significance. In Section 1 we have already mentioned the occurrence of torus knots in chemical structures, such as in DNA. We also recall their role in the analysis of turbulent motion.

Another interesting manifestation of chirality related to torus curves is the simultaneous occurrence of magnetic dipole and magnetic anapole moments in nuclei and in atoms in which parity is violated. The anapole moment arises through a toroidal electric current which in the presence of parity violation becomes a helical toroidal current $[17,18]$, corresponding to $(p, 1)$ torus curves. Furthermore, the analysis of the electronic probability current in a parityviolated atom leads to a representation related to $(1, q)$ torus curves $[18,19]$.

In conclusion, the analysis of the chirality of space curves by the method suggested in this note for torus curves, should be of general interest in the interpretation of parity breaking in a number of physical and chemical phenomena.

\section{References}

[1] G. Gilat, J. Phys. A: Math. Gen. 22 (1989) L545.

[2] A.B. Buda and K. Mislow, J. Am. Chem. Soc. 114 (1992) 6006.

[3] H. Zabrodsky and D. Avnir, J. Am. Chem. Soc. 117 (1995) 462. 
[4] C. Liang and K. Mislow, J. Am. Chem. Soc. 116 (1994) 3588.

[5] O. Safarowsky, M. Nieger, R. Fröhlich and F. Vögtle, Angew. Chem. 112 (2000) 1699.

[6] L. Olavarrieta, M.L. Martínez-Robles, P. Hernández, D.B. Krimer and J.B. Schvartzman, Mol. Microbiol. 46 (2002) 699.

[7] J. Boeckmann and G. Schill, Tetrahedron 30 (1974) 1945.

[8] C. Liang, C. Cerf and K. Mislow, J. Math. Chem. 19 (1996) 241.

[9] E. Flapan, When Topology Meets Chemistry-A Topological Look at Molecular Chirality (Cambridge University Press, Cambridge, 2000).

[10] H.K. Moffatt and R.L. Ricca, Proc. R. Soc. Lond. A 439 (1992) 411.

[11] R.L. Ricca and M.A. Berger, Physics Today 49(12) (1996) 28.

[12] L. van Driel-Gesztelyi, P. Démoulin and C.H. Mandrini (2003), http://www.solaire.obspm.fr/ demoulin/03/review_H_cospar.pdf.

[13] E.W. Weisstein, CRC Concise Encyclopedia of Mathematics (CRC Press, Boca Raton, 1999).

[14] A. Belyaev (2004), http://www.mpi-sb.mpg.de/ belyaev/gm04.

[15] JavaView, http://www.javaview.de/vgp/tutor/torusknot/PaTorusKnot.html.

[16] D. Bar-Natan, The Knot Atlas (2005), http://www.math.toronto.edu/ drorbn/KAtlas/Torus Knots/.

[17] C.E. Wieman (2003), http://www.unifr.ch/physics/frap/3cycle/Lecture-3.pdf.

[18] D.N. Stacey, Physica Scripta T40 (1992) 15.

[19] R.A. Hegstrom, J.P. Chamberlain, K. Seto and R.G. Watson, Am. J. Phys. 56 (1988) 1086. 